\title{
The Common Causes of Irritable Bowel Syndrome (IBS) in Northern Saudi Arabia
}

\author{
Saleh Hadi Alharbi \\ Faculty of Medicine, Al-Imam Mohammad Ibn Saud Islamic University, Riyadh, Kingdom of Saudi Arabia \\ Email: hussaingad5@gmail.com
}

How to cite this paper: Alharbi, S.H. (2019) The Common Causes of Irritable Bowel Syndrome (IBS) in Northern Saudi Arabia. International Journal of Clinical Medicine, 10, 91-100.

https://doi.org/10.4236/ijcm.2019.102009

Received: January 30, 2019

Accepted: February 19, 2019

Published: February 22, 2019

Copyright (c) 2019 by author(s) and Scientific Research Publishing Inc. This work is licensed under the Creative Commons Attribution International License (CC BY 4.0).

http://creativecommons.org/licenses/by/4.0/

\section{(c) (i) Open Access}

\begin{abstract}
Background: Several psychological factors have been linked to the etiology of irritable bowel syndrome (IBS). The aim of the present study was to assess the burden of psychological factors (anxiety, depression, and working status) in the etiology of IBS in Northern Saudi Arabia. Methodology: Data regarding IBS were attained from 930 Saudi volunteers existing in the city of Hail, Northern Saudi Arabia. A purposeful questionnaire was developed and used to obtain the required data. Each questionnaire was completed by a medical student in the course of the interview. Results: IBS symptoms were recognized in $46 \%$ of the study population of whom $38 \%$ were males and $62 \%$ were females. The risk of anxiety as a risk for IBS was statistically significant. The relative risk and $95 \%$ confidence interval $(95 \% \mathrm{CI})=\mathrm{RR}(95 \% \mathrm{CI})=1.4801$ (1.2608 to 1.7376), $\mathrm{P}<0.0001$. The risk of emotional stress as a risk for IBS was statistically significant. The relative risk and $95 \%$ confidence interval $(95 \% \mathrm{CI})=\mathrm{RR}(95 \% \mathrm{CI})=1.5337(1.2918$ to 1.8211$), \mathrm{P}<0.0001$. Conclusion: IBS and IBS related symptoms are prevalent in Northern Saudi Arabia. IBS symptoms are significantly associated with anxiety, depression and to a lesser extent may be linked to occupation and daily working hours.
\end{abstract}

\section{Keywords}

Irritable Bowel Syndrome, Anxiety, Depression, Emotional Stress, Working Hours, Saudi Arabia

\section{Introduction}

Irritable bowel syndrome (IBS) is a chronic disorder that can considerably decrease the quality of life and work efficiency [1]. IBS is a public medical complaint that significantly alters the patient quality of life and makes a series of diagnostic and treatment challenges [2]. IBS upsets $7 \%$ to $21 \%$ of the universal population. The prevalence of IBS fluctuates amongst nations, as well as criteria 
used to describe its manifestation. Females are at somewhat greater risk for IBS than males [3]. In Saudi Arabia, studies conducted on medical students and interns in Jeddah reported a prevalence of IBS of $31.8 \%$ [4].

The diagnosis of IBS depends on the documentation of characteristic symptoms and the barring of other organic illnesses. Managing of patients with IBS is enhanced by a personalized, complete way that holds dietary, lifestyle, medical, and behavioral interventions [1]. Investigation regularity in patients with IBS is intensely connected to demographic and clinical characteristics, mainly comorbid conditions associated with IBS. The existence of shared overlapping comorbid conditions should upsurge clinicians' sureness in making the diagnosis of IBS, thus limiting redundant investigation and decreasing healthcare expenditures [5].

Psychological factors have been conspicuously involved in the causativeness as well as upkeep of IBS. The high prevalence of psychiatric comorbidities such as anxiety and depression in IBS delivers evidence in consideration of appropriate screening for these conditions in gastrointestinal clinics. Identification and treatment for these comorbidities can enhance the quality of life as well as whole consequences [6]. For patients with IBS, mental illness, mainly depression and/or anxiety, leads to an additional subordinate quality of life [7]. As several studies reported that IBS is associated with increased psychological distress and mental comorbidity, like major depressive disorder and generalized anxiety disorder [8], our aim in the present study was to assess the burden of psychological factors (anxiety, depression, and working periods) in the etiology of IBS in Northern Saudi Arabia.

\section{Materials and Methods}

In this community based cross-sectional survey, data about IBS were obtained from 930 Saudi volunteers living in the city of Hail, Northern Saudi Arabia. Participants were randomly selected by simple random regardless of age, gender, education level, occupation. Randomization was done separately for each gender (taking numbers of respondents as, 1, 3, 5, 7, etc.). About 1300 persons were recruited and 930 (71.5\%) persons have responded.

Data were collected during the period from October 2018 to January 2019.

A purposeful questionnaire was designed and used for obtaining the necessary data. Each questionnaire was filled by a medical student during the interview. The following information was obtained from each participant: age, sex, and education level, anxiety, anxiety frequency, depression, depression frequency, emotional stress, and working hours.

IBS symptoms were identified based on Rome III criteria.

\subsection{Data Analysis}

Statistical Package for Social Sciences (version 16) was used for analysis and to perform a Pearson Chi-square test for statistical significance (P value). The 95\% confidence level and confidence intervals were used. A p value less than 0.05 was 
considered statistically significant.

\subsection{Ethical Consent}

Each participant was asked to sign a written ethical consent during the questionnaire's interview. The informed ethical consent form was designed and approved by the ethical committee of the College of Medicine (University of Hail, Saudi Arabia) Research Board.

\section{Results}

The present study, involved 930 Saudi volunteers, their ages ranging from 18 to 78 years with a mean age of $34 \pm 13.7$ years. Out of 930 participants, 395/930 (42\%) were males and 535/930 (58\%) females. Greatest numbers of participants were found at the age groups $21-29(\mathrm{n}=360), \geq 50(\mathrm{n}=157)$, and $40-49$ years $(\mathrm{n}=151)$. Males were predominantly seen at the age groups $\geq 50(\mathrm{n}=115), 40$ $49(\mathrm{n}=97)$ and 21-29 years $(\mathrm{n}=92)$. Females were predominantly at $21-29(\mathrm{n}=$ 268), $\leq 20(n=108)$, and $30-39$ years $(n=63)$, as indicated in Table 1, Figure 1.

The majority of the study population were found with university level of education ( $\mathrm{n}=523)$, and secondary education $(\mathrm{n}=245)$, as shown in Table 1, Figure 1.

IBS symptoms were identified in $46 \%$ of the study population of whom (38\%) were males and $(62 \%)$ were females.

Anxiety was stated by 514 participants of whom 283 (55\%) were found with IBS symptoms. The risk of anxiety as a risk for IBS was statistically significant. The relative risk and 95\% confidence interval $(95 \% \mathrm{CI})=\mathrm{RR}(95 \% \mathrm{CI})=1.4801$ (1.2608 to 1.7376$), \mathrm{P}<0.0001$. Most patients experienced anxiety once daily $164 / 252(65 \%)$ and the remaining 35\% reported anxiety 2 to 3 times daily, as indicated in Table 2, Figure 2.

Table 1. Distribution of the study population by gender, age, and education.

\begin{tabular}{cccc}
\hline Variable & Males & Females & Total \\
\hline Age & 13 & 108 & 121 \\
$\leq 20$ years & 92 & 268 & 360 \\
$21-29$ & 78 & 63 & 141 \\
$30-39$ & 97 & 54 & 151 \\
$40-49$ & 115 & 42 & 157 \\
$\geq 50$ & 395 & 535 & 930 \\
Total & & & \\
Education & 33 & 27 & 60 \\
Illiterate & 27 & 38 & 65 \\
Basic & 125 & 120 & 245 \\
Secondary & 199 & 324 & 523 \\
University & 11 & 26 & 37 \\
Post-university & 395 & 535 & 930 \\
Total & & & \\
\hline
\end{tabular}




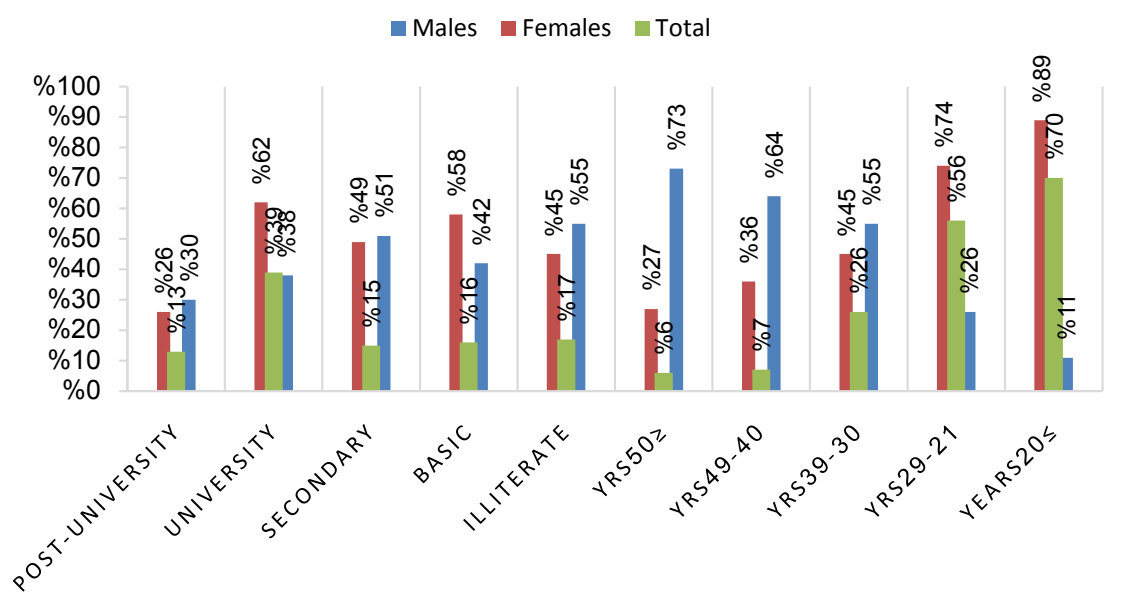

Figure 1. Description of the study population by gender, age, and education.

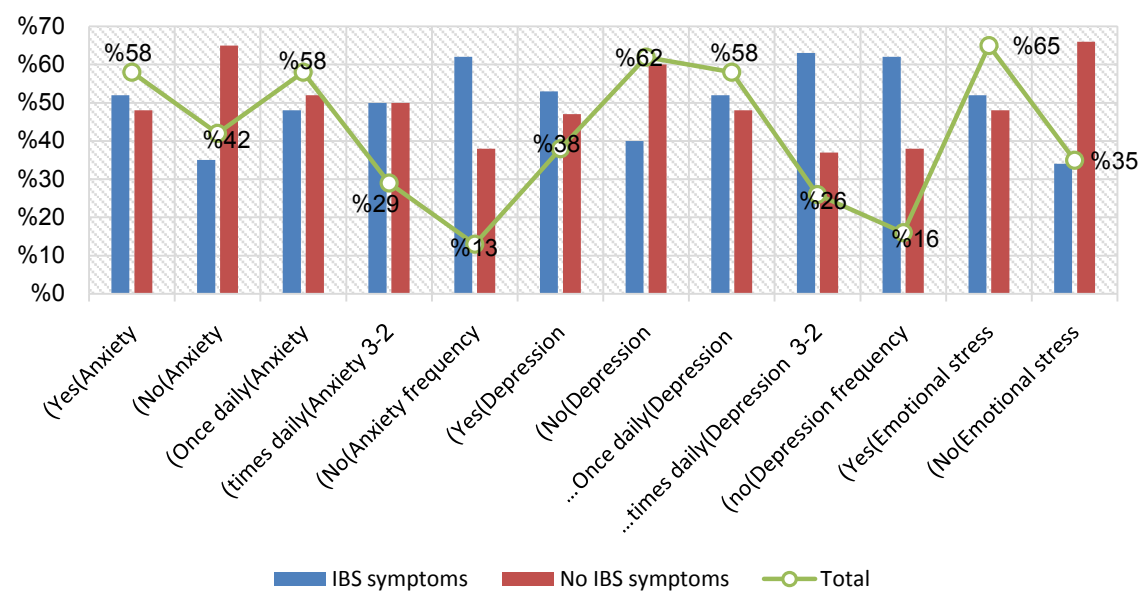

Figure 2. IBS symptoms by psychological causes.

Table 2. Distribution of the study population by IBS symptoms and psychological causes.

\begin{tabular}{cccc}
\hline Variable & IBS symptoms & No IBS symptoms & Total \\
\hline Anxiety & 283 & 258 & 541 \\
Yes & 129 & 236 & 365 \\
No & 412 & 494 & 906 \\
Total & & & \\
Anxiety frequency & 164 & 159 & 343 \\
Once daily & 88 & 86 & 174 \\
2 - 3 times daily & 48 & 29 & 77 \\
No & 320 & 274 & 594 \\
Total & 177 & & \\
Depression & 223 & 160 & 337 \\
Yes & 400 & 329 & 552 \\
No & & 489 & 889 \\
Total & & &
\end{tabular}




\section{Continued}

\begin{tabular}{cccc}
\hline \multicolumn{2}{c}{ Depression frequency } & & \\
Once daily & 116 & 115 & 223 \\
$2-3$ times a day & 62 & 37 & 99 \\
no & 39 & 24 & 63 \\
Total & 217 & 176 & 393 \\
Emotional stress & & & \\
Yes & 310 & 287 & 597 \\
No & 108 & 211 & 319 \\
Total & 418 & 498 & 916 \\
\hline
\end{tabular}

Depression was indicated by 337 participants of whom 177 (52.5\%) were found with IBS symptoms. The risk of depression as a risk for IBS was statistically significant. The RR ( $95 \% \mathrm{CI})=1.3001$ (1.1264 to 1.5006$), \mathrm{P}=0.0003$. Most patients experienced depression once daily $116 / 178$ (65\%) and the remaining $35 \%$ reported depression 2 to 3 times daily, as indicated in Table 2, Figure 2.

Emotional stress was stated by 597 participants of whom 310 (52\%) were found with IBS symptoms. The risk of emotional stress as a risk for IBS was statistically significant. The relative risk and $95 \%$ confidence interval $(95 \% \mathrm{CI})=$ $\mathrm{RR}(95 \% \mathrm{CI})=1.5337$ (1.2918 to 1.8211$), \mathrm{P}<0.0001$, as indicated in Table 2, Figure 2.

IBS symptoms were identified in 96/167 (57.5\%), 45/81 (55.6\%), 94/261 (36\%), 144/328 (44\%), 21/36 (58.3\%) and 17/41 (41.5\%) of the jobless, employers, self-employed, governmental jobs, students, and business, respectively, as indicated in Table 3, Figure 3.

With regard to the working hours, the majority of IBS symptoms were encountered among those working 6 - 8 hours/day followed by $4-6$ hours, constituting $353 / 728$ (48.5\%) and 212/728 (29\%), in this order. However, within the entire working hour's groups, the proportions of IBS increased with increasing of working hours, but it didn't show any statistically significant differences, as shown in Table 3, Figure 3.

\section{Discussion}

The burden of IBS is increasing in Saudi Arabia amongst different population settings, which might be associated with daily life tension. The prevalence of anxiety and depression and other psychological factors represent major IBS predictors in line with socioeconomic characteristics of the Saudi community.

The present study highlighted the roles of anxiety, depression, working hours, and occupation in the etiology of IBS. The study included diverse population settings in term of age, sex, education, and occupation. IBS symptoms were identified in $46 \%$ of the study population of whom (38\%) were males and $(62 \%)$ were females. Several published data indicated that IBS is a significant problem 
Table 3. Distribution of the study population by IBS symptoms, occupation and working hours.

\begin{tabular}{cccc}
\hline Variable & IBS symptoms & No IBS symptoms & Total \\
\hline Occupation & 96 & 71 & 167 \\
Jobless & 45 & 36 & 81 \\
Employer & 94 & 167 & 261 \\
Self-employed & 144 & 184 & 328 \\
Governmental jobs & 21 & 15 & 36 \\
Students & 17 & 24 & 41 \\
Business & 417 & 497 & 914 \\
Total & & & \\
Working hours & 40 & 35 & 75 \\
$<4$ hours/day & 115 & 97 & 212 \\
$4-6$ & 114 & 239 & 353 \\
6 - 8 & 32 & 32 & 64 \\
$8-10$ & 10 & 11 & 24 \\
$>10$ hours & 311 & 414 & 728 \\
Total & & & \\
\hline
\end{tabular}

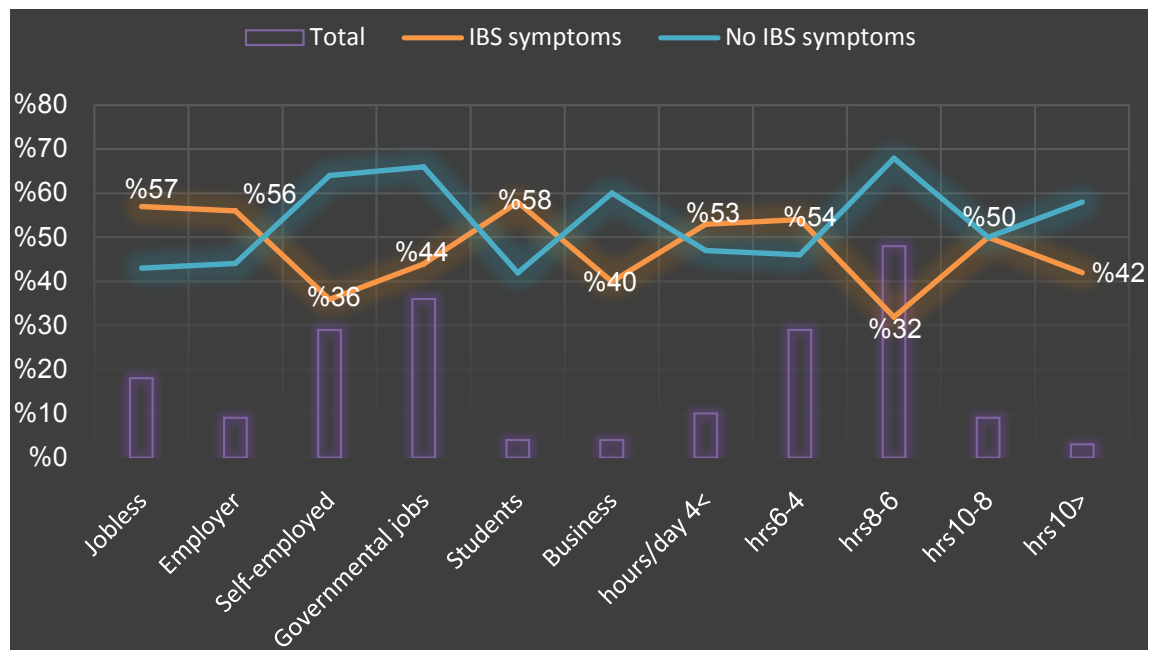

Figure 3. IBS symptoms by occupation and working hours.

among females compared to males. Female sex predisposes children and adolescents to develop IBS [9]. The reported prevalence rates of IBS among Saudi medical students and nurses were ranging from $14.4 \%$ [10] to $31.8 \%$ [11]. However, the high percentage of IBS in the present series may be attributed to the fact that, the present study considered any IBS related symptoms.

The findings of the present study showed that anxiety a strong risk for IBS. The RR $(95 \% \mathrm{CI})=1.4801$ (1.2608 to 1.7376), $\mathrm{P}<0.0001$. Moreover, depression was also statistically significant risk associated with IBS. The RR $(95 \% \mathrm{CI})=$ 
1.3001 (1.1264 to 1.5006$), P=0.0003$. In a study from Saudi Arabia to determine the prevalence, severity, and predictors of IBS among nurses, found that morbid anxiety is one of the major predictors of IBS [10]. A study from Saudi Arabia reported a high prevalence of IBS existing among medical students and interns. Female gender, morbid anxiety, living in the school dormitory, emotional stress, and higher educational level (grade) were the predictors of IBS [11]. Another study investigated medical students provided evidence that as medical students of the higher year of their under graduation were having a higher level of anxiety which leads to IBS [12]. In a systemic review involved 16 studies related to IBS, the prevalence of IBS among medical students ranged from $9.3 \%$ to $35.5 \%$. The relatively high prevalence among medical students may be attributed to their special stressful learning environment. Some studies found that female gender, family history of IBS, psychiatric stress, anxiety, depression, infections, dietary factors, and sleep disorders were associated with IBS [13]. Moreover, in the present study, the frequencies of anxiety and depression were relatively lower (once per day), which may indicate relatively moderate IBS symptoms.

The risk of emotional stress as a risk for IBS was statistically significant. The relative risk and $95 \%$ confidence interval $(95 \% \mathrm{CI})=\mathrm{RR}(95 \% \mathrm{CI})=1.5337$ (1.2918 to 1.8211 ), $\mathrm{P}<0.0001$. Our emotional situation can have several magnitudes on our somatic health and well-being. Negative emotions such as anxiety play a chief role in gut functioning because of the bidirectional communications between the gut and brain, known as, the brain-gut axis [14]. In a study examined the responses to emotional stress in IBS patients, it delivers evidence that there is a definite change in stress responses in IBS patients, but no total overstated stress response [15]. The biopsychosocial model applied to the understanding of IBS pathophysiology proposed that psychosocial factors, interacting with peripheral/central neuroendocrine and immune alterations, may encourage symptoms of IBS, modify symptom severity, influence illness experience and quality of life, and disturbing outcome. An earlier study has proposed that negative emotions and attitudes have harmful effects on health, and a number of behavioral and biological mechanisms could trigger these links [16]. However, the prevalence of psychiatric conditions in IBS patients differs in diverse cultures [17].

In the present study, IBS symptoms were frequently seen in all occupational settings. However, some of these occupations are stressful. Stress has been involved as contributing to the initiation and exacerbation of bowel and discomfort symptoms in patients with IBS [18]. The Pressure Management Indicator (PMI) is a validated questionnaire to analyze all aspects of occupational stress-a model encompassing causes of stress, the mean of coping, the personality, and the consequential effects of the communication between these 3 components. The level of occupational stress was higher in IBS patients compared to healthy subjects (socio-professional stress effects were lower in IBS patients) and associated with IL-6 levels [19]. IBS was more common in healthcare professionals than in the control group. Healthcare workers are more disposed to IBS because 
of their stressful working environment [20]. Well-being at work has been revealed to be predisposed by job features and personal variances in coping styles. Workplace anxieties, intrinsic and extrinsic effort, and negative coping and attributional behaviors were connected to high levels of depression and anxiety and low job satisfaction [21].

In the present study, higher proportions of IBS symptoms were observed among those with prolonged working hours or those with shorter working hours. There is a lack of data regarding this issue, which can be an aspect for future research.

The limitation of the present study includes its cross sectional setting as well as utility of only parts of the IBS related risk factors.

\section{Conclusion}

IBS and IBS related symptoms are prevalent in Northern Saudi Arabia. IBS symptoms are significantly associated with anxiety, depression and to a lesser extent may be linked to occupation and daily working hours. More studies in this context are rendered important to provide sufficient evidence for intervention and control.

\section{Acknowledgements}

The author would like to cordially thank Prof. Hussain Gadelkarim Ahmed for his meticulous revision of the manuscript. Author would like to thank students at the College of Medicine, University of Hail for their help in data collection.

\section{Conflicts of Interest}

The author declares no conflicts of interest regarding the publication of this paper.

\section{References}

[1] Chey, W.D., Kurlander, J. and Eswaran, S. (2015) Irritable Bowel Syndrome: A Clinical Review. JAMA, 3, 949-958. https://doi.org/10.1001/jama.2015.0954

[2] Defrees, D.N. and Bailey, J. (2017) Irritable Bowel Syndrome: Epidemiology, Pathophysiology, Diagnosis, and Treatment. Prim Care, 44, 655-671. https://doi.org/10.1016/j.pop.2017.07.009

[3] Lovell, R.M. and Ford, A.C. (2012) Global Prevalence of and Risk Factors for Irritable Bowel Syndrome: A Meta-Analysis. Clinical Gastroenterology and Hepatology, 10, 712-721.e4. https://doi.org/10.1016/j.cgh.2012.02.029

[4] Ibrahim, N.K., Battarjee, W.F. and Almehmadi, S.A. (2013) Prevalence and Predictors of Irritable Bowel Syndrome among Medical Students and Interns in King Abdulaziz University, Jeddah. Libyan Journal of Medical Sciences, 8, Article ID: 21287. https://doi.org/10.3402/ljm.v8i0.21287

[5] Lacy, B., Ayyagari, R., Guerin, A., Lopez, A., Shi, S. and Luo, M. (2019) Factors Associated with More Frequent Diagnostic Tests and Procedures in Patients with Irritable Bowel Syndrome. Therapeutic Advances in Gastroenterology, 12, Article ID: 1756284818818326. 
[6] Banerjee, A., Sarkhel, S., Sarkar, R. and Dhali, G.K. (2017) Anxiety and Depression in Irritable Bowel Syndrome. Indian Journal of Psychological Medicine, 39, 741-745. https://doi.org/10.4103/IJPSYM.IJPSYM_46_17

[7] Akama, F., Mikami, K., Watanabe, N., Kimoto, K., Yamamoto, K. and Matsumoto, H. (2018) Efficacy of Mirtazapine on Irritable Bowel Syndrome with Anxiety and Depression: A Case Study. Journal of Nippon Medical School, 85, 330-333. https://doi.org/10.1272/jnms.JNMS.2018_85-53

[8] Gajdos, P. and Rigó, A. (2018) Irritable Bowel Syndrome: Comorbid Psychiatric Disorders and Psychological Treatment Options. Orvosi Hetilap, 159, 2115-2121. https://doi.org/10.1556/650.2018.31247

[9] Devanarayana, N.M., Rajindrajith, S., Pathmeswaran, A., Abegunasekara, C., Gunawardena, N.K. and Benninga, M.A. (2015) Epidemiology of Irritable Bowel Syndrome in Children and Adolescents in Asia. Journal of Pediatric Gastroenterology and Nutrition, 60, 792-798. https://doi.org/10.1097/MPG.0000000000000714

[10] Ibrahim, N.K., Al-Bloushy, R., Sait, S.H., Al-Azhary, H.W., Bar, N.H. and Mirdad, G.A. (2016) Irritable Bowel Syndrome among Nurses Working in King Abdulaziz University Hospital, Jeddah, Saudi Arabia. Libyan Journal of Medical Sciences, 11, Article ID: 30866. https://doi.org/10.3402/ljm.v11.30866

[11] Ibrahim, N.K., Battarjee, W.F. and Almehmadi, S.A. (2013) Prevalence and Predictors of Irritable Bowel Syndrome among Medical Students and Interns in King Abdulaziz University, Jeddah. Libyan Journal of Medical Sciences, 8, Article ID: 21287. https://doi.org/10.3402/ljm.v8i0.21287

[12] Alaqeel, M.K., Alowaimer, N.A., Alonezan, A.F., Almegbel, N.Y. and Alaujan, F.Y. (2017) Prevalence of Irritable Bowel Syndrome and its Association with Anxiety among Medical Students at King Saud bin Abdulaziz University for Health Sciences in Riyadh. Pakistan Journal of Medical Sciences, 33, 33-36. https://doi.org/10.12669/pjms.331.12572

[13] Ibrahim, N.K. (2016) A Systematic Review of the Prevalence and Risk Factors of Irritable Bowel Syndrome among Medical Students. The Turkish Journal of Gastroenterology, 27, 10-16. https://doi.org/10.5152/tjg.2015.150333

[14] Pellissier, S. and Bonaz, B. (2017) The Place of Stress and Emotions in the Irritable Bowel Syndrome. Vitamins \& Hormones, 103, 327-354.

https://doi.org/10.1016/bs.vh.2016.09.005

[15] Bach, D.R., Erdmann, G., Schmidtmann, M. and Mönnikes, H. (2006) Emotional Stress Reactivity in Irritable Bowel Syndrome. European Journal of Gastroenterology \& Hepatology, 18, 629-636. https://doi.org/10.1097/00042737-200606000-00009

[16] Muscatello, M.R., Bruno, A., Scimeca, G., Pandolfo, G. and Zoccali, R.A. (2014) Role of Negative Affects in Pathophysiology and Clinical Expression of Irritable Bowel Syndrome. World Journal of Gastroenterology, 20, 7570-7586. https://doi.org/10.3748/wjg.v20.i24.7570

[17] Modabbernia, M.J., Mansour-Ghanaei, F., Imani, A., Mirsafa-Moghaddam, S.-A., et al. (2012) Anxiety-Depressive Disorders among Irritable Bowel Syndrome Patients in Guilan, Iran. BMC Research Notes, 5, 112.

https://doi.org/10.1186/1756-0500-5-112

[18] Hertig, V.L., Cain, K.C., Jarrett, M.E., Burr, R.L. and Heitkemper, M.M. (2007) Daily Stress and Gastrointestinal Symptoms in Women with Irritable Bowel Syndrome. Nursing Research, 56, 399-406. https://doi.org/10.1097/01.NNR.0000299855.60053.88

[19] Popa, S.L., Leucuta, D.C. and Dumitrascu, D.L. (2018) Pressure Management as an 
Occupational Stress Risk Factor in Irritable Bowel Syndrome: A Cross-Sectional Study. Medicine, 97, e13562. https://doi.org/10.1097/MD.0000000000013562

[20] Tosun, O., Dabak, R., Sargin, M., Dolapcioglu, C. and Ahishali, E. (2016) Frequency of Irritable Bowel Syndrome among Healthcare Personnel. Gastroenterology Nursing, 39, 227-231. https://doi.org/10.1097/SGA.0000000000000188

[21] Mark, G. and Smith, A.P. (2012) Effects of Occupational Stress, Job Characteristics, Coping, and Attributional Style on the Mental Health and Job Satisfaction of University Employees. Anxiety, Stress \& Coping, 25, 63-78.

https://doi.org/10.1080/10615806.2010.548088 